\title{
Effect of Web-based Clinical Decision Support Systems on Adherence to Venous Thromboembolism Prophylaxis guideline among ICU Nonsurgical Patients: A Prospective Before and After Study
}

Mehrdad Karajizadeh

Shiraz University of Medical Sciences

\section{Farid Zand}

Shiraz University of Medical Sciences

Roxana Sharifian ( $\nabla$ sharifianr@sums.ac.ir)

Shiraz University of Medical Sciences

Afsaneh Vazin

Shiraz University of Medical Sciences

Najmeh Bayati

Shiraz University of Medical Sciences

\section{Research Article}

Keywords: Clinical Decision Support Systems, Prophylaxis, Venous Thromboembolism, Intensive Care Unit, Nonsurgical, Recommendation System

Posted Date: September 7th, 2021

DOl: https://doi.org/10.21203/rs.3.rs-842416/v1

License: (c) (i) This work is licensed under a Creative Commons Attribution 4.0 International License.

Read Full License 


\section{Abstract}

Background and objective: There is a gap between expert recommendations and clinical practice in (Venous Thromboembolism) VTE prophylaxis among nonsurgical patients worldwide. Rate of adherence to evidence-based practice is inadequate in the nonsurgical population. Therefore, this study aimed to determine The effect of Clinical Decision Support Systems(CDSS) on the use of the appropriate VTE Prophylaxis in Nonsurgical Patients in the Intensive Care Unit (ICU).

Method: We conducted a cross-sectional study (pre and post-implementation CDSS for recommendation VTE prophylaxis order set) to analyze the effect of the CDSS within CPOE on the appropriate VTE prophylaxis in three ICUs of the Nemazee hospital (before intervention from 20 April 2020, to 21 November 2020 and post-intervention duration form 7 April 2021, to 9 July 2021). The pre-intervention and post-intervention phase samples comprised 175 and 27 patients, respectively. P-value is less than 0.05 was considered a significant level. All statistical analysis was performed by SPSS version 24 .

Results: Adherence to VTE prophylaxis guidelines after introduced CDSS for recommendation VTE prophylaxis within CPOE system in nonsurgical patients in ICUs increase from $48.6 \%$ to $77.8 \%$ (pvalue $<01)$. However, mortality rate (pre-intervention $13.80 \%$ vs post-intervention $14.80 \%(p$-value $=0.88)$ ) and means of length of stay (pre-intervention 13.66 vs post intervention13.63( $p$-value=0.49)) in ICU have not significantly change after introduced CDSS for recommendation VTE prophylaxis order sets.

Conclusion: The results indicate that the CDSS for recommendation VTE prophylaxis within CPOE improves adherence to VTE prophylaxis in nonsurgical patients at ICUs, which assist provider to select the most tailored VTE prophylaxis. Further study needs to evaluate implemented CDSS for recommendation VTE prophylaxis in nonsurgical patients at a province and national level.

\section{Background}

Venous thromboembolism (VTE) is one of the most common complications in nonsurgical critical patients. Incidence of VTE hospitalized ill medical patients have an eight-time higher than the general population(1). The incidence of VTE in ICU is up to $60 \%(2,3)$.

VTE developing in ICU increases hospital length of stay and mortality(4). Annual hospitalization costs of nonsurgical patients with a diagnosis of VTE are from 13.4 to 27.3 billion(5). Although some VTE events are unpreventable, several studies have shown that appropriate VTE prophylaxis in high-risk critical patients reduces the risk of VTE and related complications(6). However, several studies have demonstrated that some hospitalized patients receive inappropriate VTE prophylaxis(7). Inappropriate VTE prophylaxis increases adverse complications(8). VTE guideline recommendations produced by the American College of Chest Physicians (ACCP) are considered to be given appropriate VTE prophylaxis in nonsurgical patients(9). 
Multi-faced interventions have been attempted to improve appropriate VTE prophylaxis in different patient populations(10). This intervention strategy includes education intervention, increasing delivery of ordered prophylaxis, reduced use of central venous catheters, enhanced mobility, Audit and feedback, pock guideline distribution, care pathway, checklists, electronic alerts in digital medical records, prescription reminder, pre-established orders, and order sets embedded VTE prevention protocol(10-14). Improving appropriate VTE prophylaxis is multifactorial. clinicians should assess patient risk for VTE and prescribe therapy appropriate for each patients' risk profile, the patient should accept the prescribed therapy, and nurses must administer the therapy as prescribed. First, an ideal, proper VTE prophylaxis process measure requires; 1-documentation of a standardized VTE risk assessment; 2-prescription of optimal, risk-appropriate VTE prophylaxis; and 3-administration of all risk-appropriate VTE prophylaxis as prescribed(15).

A systematic review and meta-analysis study have indicated that introducing CDSS increases VTEappropriate prophylaxis in surgical patients(16). Mandatory computerized CDS tool for VTE prophylaxis in trauma patients significantly improves compliance with VTE prophylaxis guidelines(17). CDSS for VTE prophylaxis in surgical patients increase compliance to the guideline but did not significantly alter VTE outcome(18). Prior knowledge-translation (KT) interventions applying "high-tech" approaches, such as electronic alerts, electronic CDSS, and computerized provider order entry(CPOE) systems, have improved compliance with clinical guidelines(19). A computerized alert system improves appropriate VTE prophylaxis and improves risk assessment documentation. However, the Adoption computerized alert system for improving VTE prophylaxis rates is uncertain(20).

there is a gap between expert recommendations and clinical practice in VTE prophylaxis in nonsurgical patients worldwide, yet $(9,21-24)$. Thus, the rate of adherence to evidence-based practice of VTE prophylaxis is inadequate in the nonsurgical population. Consequently, the rate of VTE in nonsurgical patients is high. Yet, hospital-acquired VTE is a global challenge(25).

CDS tools improve evidence-based medicine to patient care(26), especially improve the implementation of VTE prophylaxis guidelines. Nevertheless, there is not research investigate the effect of CDS for recommendation prophylaxis on appropriate VTE prophylaxis in nonsurgical ICU patients in Iranian hospital. despite CDS clear advantages in the world, the effects of CDSS within the CPOE system on appropriate prophylaxis for nonsurgical ICU patients remain uncertain. In addition, our previous study indicates that CDS for recommendation VTE prophylaxis has reached high priority to implementation within CPOE in ICU, based on CDS committee consensus in Iran(27). Still, there is a gap between expert recommendations and clinical practice in VTE prophylaxis in a nonsurgical ICU patient in Iranian hospital. Therefore, we conducted a study aimed at assessing the impact of real-time computerized CDSS on the use of appropriate prophylaxis for VTE among nonsurgical ICU patients in a southern Iran.

\section{Methods}


All methods were conducted accordance with (Strengthening the Reporting of Observational studies in Epidemiology) STROPE guidelines for this study. This is a cross-sectional study to assess the effect of a real-time CDSS for recommendation VTE prophylaxis within CPOE on adherence to VTE prophylaxis guideline in nonsurgical patients. this study was conducted at the three ICUs (General ICU, Central ICU, and Emergency ICU) of Nemazee hospital, which is located in Shiraz, and is the largest academic hospital in southern Iran. Data related to before intervention have been collected from April 20, 2020, to 21 November 2020 and post-intervention have been collected form 7 April 2021, to 9 July 2021). It is a teaching hospital, with 850 beds. Three ICUs of Nemazee hospital uses the homegrown electronic medical record (EMR) with a CPOE system since 2015(28). The Homegrown EMR implemented in Nemazee hospital has not equipment the VTE prophylaxis CDSS. In three ICUs serviced by one medical team, with similar services. All ICUs was administered by intensivists with a closed system. The inclusion criteria included nonsurgical patients aged over 18 years and having been hospitalized in General ICU, Central ICU, and emergency ICU of Nemazee hospital. another hand pregnancy, and surgical patients and patients' current use of anticoagulation were excluded because surgical and pregnancy populations were used different guidelines.

For Real-Time computerized clinical decision support systems on the use of Appropriate Prophylaxis for VTE in nonsurgical patients' intervention

\section{First step:}

modified VTE prophylaxis guidelines nonsurgical for use at ICU in Iran. Intensivists (three individuals), clinical pharmacy (one individual), and health informatics (two individuals) from Shiraz University of Medical Science(SUMS) were invited to attend the panel. In three sessions panel members discuss VTE prophylaxis guideline use in Iran. VTE risk assessment model and appropriate prophylaxis in nonsurgical patients at ICU in Iran country and contraindication of VTE prophylaxis was modified and established by this panel. Local VTE prophylaxis guidelines based on American College of Chest Physicians (ACCP) prophylaxis in nonsurgical patients are being in the supplementary file. Appendix 1.

\section{The second step}

design, development, and implement CDS for recommendation VTE prophylaxis within the CPOE system. Common UML (unified model language) diagrams included use case, activity diagram, and sequence diagram were designed for the VTE prophylaxis CDSS (as can be seen in UML diagrams in supplementary file2 appendix 2). The knowledge base of VTE prophylaxis CDSS in nonsurgical patient consists of 26 rule sets. After that, the VTE prophylaxis CDSS was developed in CPOE (as can be seen in Screen of this CDSS in supplementary file3 appendix 3). In the next step, chairman of the ICU and CDSS committee in the hospital was assessment and tested the VTE prophylaxis CDSS. All major bugs or changes in functionality or content have been fixed after the testing system. After approval VTE prophylaxis system by the CDS committee, all end-users (clinicians) have been trained to use the VTE 
prophylaxis CDSS in class and one by one. Finally, the VTE prophylaxis CDSS has been installed in three ICUs of Nemazee hospitals. The physician has completed the VTE risk factors checklist and contradiction document for the nonsurgical patient, then CDSS based on VTE risk score, contraindication VTE prophylaxis, patient weight, and renal functions recommended VTE prophylaxis order sets. The content of VTE prophylaxis CDS was changed by consensus of the CDS committee includes three ICU attending, a clinical pharmacist, and two health information management specialists. The unexpected bug in the VTE prophylaxis CDSS resolves each day.

\section{The third step}

The researcher's measurement clinician adherence to VTE prophylaxis guidelines in nonsurgical patients after implemented VTE prophylaxis CDS. The pharmacist in the research team evaluated adherence to VTE prophylaxis guidelines in nonsurgical patients.

A VTE risk assessment model for hospitalized nonsurgical patients, the Padua Score, has been established and recommended using the 2012 ACCP evidence-based clinical practice guideline for VTE prevention in nonsurgical patients (9). This tool includes 11 risk factors including age, Body Mass Index(BMI), history of VTE, a surgical procedure during present hospitalization, acute myocardial infarction or ischemic stroke, presence of malignancy, heart or respiratory failure, and hormonal treatment. The score of each VTE risk factor in nonsurgical patients recommended VTE prophylaxis based on VTE score, and contraindication of each VTE prophylaxis is presented in the supplementary file. appendix 1. A Padua Prediction Score was calculated for each patient. The patient was identified as high risk if they had a calculated score $>3$.

Variables that were collected from the patients' interviews and completed from the medical file for all patients from the adult ICU included demographics (age, and sex), ICU length of stay, mortality in ICU, and risk factors of VTE.

For the baseline period, researchers reviewed each patient's medical record to collect the following VTErelated variable: provider documentation of Padua risk stratification, patient VTE risk factors, contraindications to pharmacological prophylaxis, and written orders for prophylaxis within $24 \mathrm{~h}$ of admission. For the post-implementation period, these variables were extracted directly from the EMR and CPOE system. Compliance with appropriate best practice VTE prophylaxis guidelines was defined as adherence to local VTE prevention algorithm. The primary outcome was appropriate VTE prophylaxis or adherence to the local VTE prophylaxis algorithm. The secondary outcome was ICU mortality and ICU length of stay.

Continuous variables are summarized with means and standard deviation, and categorical variables are summarized with numbers and proportions. Adherence to VTE prophylaxis guidelines in the group without VTE prophylaxis CDS compared to the group using CDSS for recommendation VTE prophylaxis, by using the 2 -sided $\mathrm{X}^{2}$ test for categorical variables and Student t-test was used to the evaluated effect 
of CDS on appropriate VTE prophylaxis and patient's outcome. Value of $\mathrm{P}<0.05$ was considered statistically significant. All statistical analysis was performed by SPSS version 24 .

\section{Results}

Two hundred two patients have participated in the study (175 individuals in the pre-implementation group, 27 individuals in the post-implementation group). Table 1 presents characteristics of patients who are admitted in ICUs in pre-intervention and post-intervention groups over study periods. The mean age of the population in the pre-intervention group was $52.61 \pm 20.21$. While the mean age of the population in the post-intervention group was $55.11 \pm 16.17$. The mean of VTE risk scores in the pre-intervention group was lower than the post-intervention group (2.59 vs 3.67). Detail of patient baseline demographic data in pre and post-implementation groups are represented in Table 1.

Table 1

baseline characteristics of patients admitted to adult ICUs of large Academic Hospital

\begin{tabular}{|lll|}
\hline Baseline data & Pre-intervention & Post-intervention \\
\hline All admission in the study period (N;\%) & $175(86.6 \%)$ & $27(13.4 \%)$ \\
\hline Age (Mean; SD) & $52.61 \pm 20.21$ & $55.11 \pm 16.17$ \\
\hline Gender (Female \%) & $75(42.90 \%)$ & $16(59.3 \%)$ \\
\hline Gender (Male \%) & $100(57.10 \%)$ & $11(40.7 \%)$ \\
\hline Weight (Mean; SD) & $72.74 \pm 16.53$ & $79.11 \pm 11.27$ \\
\hline Height (Mean; SD) & $168.72 \pm 8.56$ & $167.89 \pm 7.17$ \\
\hline Creatinine(Mean; SD) & $1.43 \pm 0.97$ & $1 / 52 \pm 0 / 84$ \\
\hline VTE risk score (Mean; SD) & $2.59 \pm 2.75$ & $3.67 \pm 2.69$ \\
\hline
\end{tabular}

Figure 1 presents the frequency of each VTE risk factor in the pre-implementation and postimplementation groups. Reduced mobility, heart and/or respiratory failure, and age over 70 years were three top common VTE risk factors in the pre and post-implementation group.

Table 2 shows the frequency of VTE prophylaxis orders pre and post-implementation CDSS for recommendation VTE prophylaxis within CPOE in Adult ICUs of a large academic hospital. The results show that most patients received heparin in the pre-intervention group (CPOE without CDSS for recommendation VTE prophylaxis) (56.9\%). While in the post-intervention group (CPOE with CDSS for recommendation VTE prophylaxis) a large percentage of patients received Enoxaparin (59.2\%). 
Table 2

Frequency VTE prophylaxis pre and post-intervention (CDSS for recommendation VTE prophylaxis within CPOE) in adult ICUs of large Academic Hospital

\begin{tabular}{|c|c|c|c|}
\hline & & Group & \\
\hline & & $\begin{array}{l}\text { Pre- } \\
\text { Implementation }\end{array}$ & $\begin{array}{l}\text { Post- } \\
\text { Implementation }\end{array}$ \\
\hline VTE & Early Ambulation & $1(0.6 \%)$ & $0(0.0 \%)$ \\
\hline & $\begin{array}{l}\text { Sequential Compression Device } \\
\text { (SCD) }\end{array}$ & $6(3.4 \%)$ & $0(0.0 \%)$ \\
\hline & Heparin 5000 units BID & $88(50.3 \%)$ & $10(37.0 \%)$ \\
\hline & Heparin 5000 units TID & $11(6.3 \%)$ & $1(3.7 \%)$ \\
\hline & Enoxaparin 40 mg QID & $9(5.1 \%)$ & $7(25.9 \%)$ \\
\hline & Enoxaparin 40 mg BID & $1(0.6 \%)$ & $0(0.0 \%)$ \\
\hline & Enoxaparin 60 mg QID & $9(5.1 \%)$ & $8(29.6 \%)$ \\
\hline & Enoxaparin 60 mg BID & $6(3.4 \%)$ & $0(0.0 \%)$ \\
\hline & None & $44(25.1 \%)$ & $0.0 \%$ \\
\hline & Enoxaparin $60 \mathrm{mg}$ QID \& SCD & $0(0.0 \%)$ & $1(3.7 \%)$ \\
\hline Total & & $175(100 \%)$ & $27(100 \%)$ \\
\hline
\end{tabular}

The results show that the proportion of patients receiving appropriate VTE prophylaxis was an increase after introduced CDSS for recommendation VTE prophylaxis within CPOE. Table 3 summarizes how the CDSS for recommendation VTE prophylaxis within CPOE effect on guideline compliance rate in ICU. The total VTE prophylaxis recommends by the CDSS was 27 smart VTE prophylaxis order sets. The percentage of no adherence to VTE prophylaxis guidelines in nonsurgical patients after implementation of CDSS for recommendation VTE prophylaxis declined from 51.4-22.2\%. In contrast, the adherence rate to VTE prophylaxis guidelines after the implementation CDSS for recommendation VTE prophylaxis increased by $29.2 \%$. Detail of performance of CDS for recommendation VTE prophylaxis is represented in Table 3. 
Table 3

Performance of a CDSS for recommendation VTE prophylaxis within CPOE in ICUs of a large academic hospital

\begin{tabular}{|lllll|}
\hline & & Group & & P- \\
\cline { 2 - 4 } & & $\begin{array}{l}\text { Pre- } \\
\text { Implementation }\end{array}$ & $\begin{array}{l}\text { Post- } \\
\text { Implementation }\end{array}$ \\
\hline $\begin{array}{l}\text { Adherence to VTE Prophylaxis Guideline in } \\
\text { nonsurgical patients }\end{array}$ & No & $90(51.4 \%)$ & $6(22.2 \%)$ & 0.005 \\
\cline { 2 - 4 } & Yes & $85(48.6 \%)$ & $21(77.8 \%)$ & \\
\hline Total & & $175(100.0 \%)$ & $27(100.0 \%)$ & \\
\hline
\end{tabular}

Table 4 represents how the CDSS for recommendation VTE prophylaxis affects the patient outcome in ICUs. The results show that the mortality rate in ICU, and length of stay in ICU was not significantly changed after implemented the CDSS for recommendation VTE prophylaxis within the CPOE system ( $p$ value $=0.88$, and 0.49 ).

Table 4

Comparison of patient outcome pre and post-intervention (CDSS for recommendation VTE prophylaxis within $\mathrm{CPOE}$ ) in ICU of large academic Hospital

\begin{tabular}{|llll|}
\hline Patient Outcome measurement & Pre-intervention & Post-intervention & P-value \\
\hline The mortality rate in ICU (N;\%) & $20(13.80 \%)$ & $4(14.80 \%)$ & P value $=0.88$ \\
\hline Length of stay in ICU (mean; SD) & $13.66 \pm 13.66$ & $13.63 \pm 9.81$ & P value $=0.49$ \\
\hline
\end{tabular}

\section{Discussion}

It was the main purpose of the study to draw attention to the effects of VTE prophylaxis CDSS for recommendation VTE prophylaxis on guideline compliance rate in nonsurgical patients at ICU. Our results describe for the first time the effects of a web-based CDSS for recommendation VTE prophylaxis within the CPOE system on guideline compliance rate in nonsurgical patients at ICU in Iran. This is an important finding in the understanding of the effects of web-based CDSS for recommendation VTE prophylaxis order set within CPOE system in nonsurgical patients at ICU of the large academic hospital in southern Iran can avert inappropriate prophylaxis prescription without effects on patient's outcomes. Also, the main finding indicates that the CDSS for recommendation VTE prophylaxis within the CPOE system improves clinician adherence to VTE prophylaxis guidelines in nonsurgical patients at ICU.

These results agreed with other studies which have shown that VTE prophylaxis CDSS for recommendation prophylaxis order set within CPOE system can improve appropriate VTE prophylaxis in nonsurgical patients at ICU, but the incidence of adherence to VTE prophylaxis guideline is a variety from country to country(29-33). In contrast to some reports in the literature, Eijgenraam and their colleagues 
found that after the introduction of VTE prophylaxis CDS did no significantly improve adherence in guideline VTE prophylaxis in nonsurgical patients(34).

This finding was quite unexpected that the CDSS for recommendation VTE prophylaxis within the CPOE system has not affected ICU mortality rate and ICU length of stay. These results consist with other studies which have shown that CDSS for recommendation VTE prophylaxis within CPOE system improves tailored prophylaxis order in nonsurgical patients without effect on patient outcome $(18,30,35)$. An important implication of this finding is that an electronic CDSS for recommendation VTE prophylaxis within CPOE System can improve appropriate prophylaxis without effects on patient outcome. Despite the CDSS improve adherence to clinical guidelines, it is maybe a negative effect on physician skills, for example, physician excessive confidence in the CDSS(36). Recommender system in medicine potential to engage users to change their behavior(37). A recent study found that drug recommender systems assist healthcare professionals in select accurate medication(38). Health recommender systems have emerged as high-tech approaches to assist clinicians to make better prevention-related decisions(38). Overall this system improves clinical management and filtered VTE prophylaxis order set based on context, as considered facilitator factor to clinician acceptance medication-related CDSS(39).

The main strength of our study was that web-based CDSS for recommendation VTE prophylaxis integrated into homegrown EMR. End-user involved in developing the content of CDSS for recommendation VTE prophylaxis engine for nonsurgical ICU patients improve successful CDSS system. these results demonstrated that CDSS for recommendation prophylaxis order engine within CPOE in nonsurgical ICU patient improve appropriate and tailored medication and VTE prophylaxis. To our best knowledge, our study was the first time to address the effect of the CDSS for recommendation VTE prophylaxis engine within CPOE in nonsurgical ICU patients of academic general hospital in Iran. However, the study had some relevant limitations. The most important limitation related to the sample size because this system was executed in a single center. In addition, this system was not integrated into EHR, because there is no EHR in Iran currently. Thus, this CDSS for recommendation VTE prophylaxis engine within CPOE cannot retrieve patient medication and disease history from EHR. Thus, medication and disease history was not uploaded to the current system at the patient admission time in the ICU of Nemazee hospital. We also have to take into account that our study could have been affected by location bias and suffered from a lack of randomized study.

Finally, the web-based CDSS for recommendation VTE prophylaxis engine within CPOE was tested and validated in ICU at a single general teaching hospital in a developing country; therefore, the implementation of the CDSS for recommendation VTE prophylaxis engine easily can be generalized to healthcare center which admitted nonsurgical patients at a province or national level.

\section{Conclusion}

This paper has clearly shown that the introduced CDSS for recommendation VTE prophylaxis within CPOE in ICUs assists providers to select the most tailored VTE prophylaxis for nonsurgical patients in ICU. 
In addition, introduced CDSS for recommendation VTE prophylaxis improve adherence to VTE prophylaxis guidelines in nonsurgical ICU patients. The findings have approved the great potential of the CDSS inappropriate treatment. Therefore, it can be useful for using the CDSS at point of care. further study needs to evaluate introduced CDSS for recommendation VTE prophylaxis in nonsurgical patients in the province and national level. Furthermore, the evaluated effect of an expert VTE prophylaxis system on appropriate VTE prophylaxis in nonsurgical patients would be of interest.

\section{Abbreviations}

VTE =Venous Thromboembolism, ICU =Intensive Care Unit, EMR =Electronic Medical Record, APACHE= Acute Physiology and Chronic Health Evaluation, SPSS= Statistical Package for the Social Sciences, ACCP =American College of Chest Physician, DVT = deep vein thrombosis, IRB =Institutional Review Board, $A H R Q=H e a l t h c a r e$ Research and Quality, CVA=Cerebral Vascular Accident, SUMS=Shiraz University of Medical Science.

\section{Declarations}

\section{Ethics approval}

The study was approved by the local Institutional Review Board and ethics committee of Shiraz University of Medical Science(SUMS) (Approval ID: IR.SUMS.REC.1398.1046). The safety and security of the CDSS are assurance. Only authorized providers can access the CDSS. all user was training for use system.

\section{Informed consent:}

Not applicable.

\section{Confidentiality}

According to the SUMS agreement, anonymity of patients is maintained by de-identification data. Only researchers or authorized personnel can access the data. To comply with SUMS and Nemazee hospital data security regulations, only research team can access patients and providers' information. SUMS and Nemazee Hospital security approved the investigation protocol.

\section{Data Availability Statement}

All relevant data are presented in the article, further required can be directed to the corresponding author(sharifianr@sums.ac.ir). 


\section{Competing Interests}

The authors declared no potential conflicts of interest to the research.

\section{Funding}

This study was supported by the Shiraz University of Medical Science(SUMS) in the context of a dissertation project (Grant Number 97-01-07-19299)

\section{Author's Contribution}

MK: conceptual and design study, data acquisition, data analysis, drafted and revision paper. FZ: contributed to conceiving and design of the study, interpretation of data, commented on drafts, and made significant revisions to the paper. AV: contributed to design of the study, interpretation of data, commented on the draft, and made significant revisions to the paper. NB: contributed to the design of the study, statistical analysis, commented on drafts, and made significant revisions to the paper. RSH: contributed to the design of the study, interpretation of data, commented on drafts, and made significant revisions to the paper.

\section{Acknowledgments}

This article was adapted from Mr. Karajizadeh's dissertation in the Health Information Management, Faculty of Management and Medical Information Sciences, Shiraz University of Medical Sciences, Shiraz, Iran. The authors would like to thank the Research Vice-Chancellor of Shiraz University of Medical Sciences for financially supporting the research (Contract No. 97-01-07-19299).

\section{References}

1. Ejaz A, Ahmed MM, Tasleem A, Rafay Khan Niazi M, Ahsraf MF, Ahmad I, et al. Thromboprophylaxis in Intensive Care Unit Patients: A Literature Review. Cureus. 2018;10(9):e3341-e.

2. Chan CM, Shorr AF. Venous Thromboembolic Disease in the Intensive Care Unit. Semin Respir Crit Care Med. 2010;31(01):039-46.

3. Cook D, Douketis J, Crowther MA, Anderson DR. The diagnosis of deep venous thrombosis and pulmonary embolism in medical-surgical intensive care unit patients. Journal of critical care. 2005;4(20):314-9.

4. Malato A, Dentali F, Siragusa S, Fabbiano F, Kagoma Y, Boddi M, et al. The impact of deep vein thrombosis in critically ill patients: a meta-analysis of major clinical outcomes. Blood Transfusion. 2015;13(4):559. 
5. Dasta JF, Pilon D, Mody SH, Lopatto J, Laliberté F, Germain G, et al. Daily Hospitalization Costs in Patients with Deep Vein Thrombosis or Pulmonary Embolism Treated with Anticoagulant Therapy. Thrombosis Research. 2015;135(2):303 - 10.

6. Skeik N, Westergard E. Recommendations for VTE Prophylaxis in Medically III Patients. Annals of Vascular Diseases. 2020:ra. 19-00115.

7. Khalili H, Dashti-Khavidaki S, Talasaz AH, Mahmoudi L, Eslami K, Tabeefar H. Is deep vein thrombosis prophylaxis appropriate in the medical wards? A clinical pharmacists' intervention study. Pharmacy World \& Science. 2010;32(5):594-600.

8. Davis JPE, O'Leary KE, Intagliata NM. Overuse of venous thromboembolism prophylaxis among hospitalized patients with liver disease. European Journal of Haematology. 2020;104(3):223-9.

9. Kahn SR, Lim W, Dunn AS, Cushman M, Dentali F, AkI EA, et al. Prevention of VTE in nonsurgical patients: antithrombotic therapy and prevention of thrombosis: American College of Chest Physicians evidence-based clinical practice guidelines. Chest. 2012;141(2):e195S-e226S.

10. Beckman MG, Abe K, Barnes K, Bartman B, Brady PJ, Hooper WC. Strategies and partnerships toward prevention of Healthcare-Associated Venous Thromboembolism. Journal of hospital medicine. 2016;11 Suppl 2(Suppl 2):S5-S7.

11. Sennesael A-L, Krug B, Sneyers B, Spinewine A. Do computerized clinical decision support systems improve the prescribing of oral anticoagulants? A systematic review. Thrombosis Research. 2020.

12. Dong $\mathrm{F}$, Zhen $\mathrm{K}$, Zhang Z, Si C, Xia J, Zhang T, et al. Effect on thromboprophylaxis among hospitalized patients using a system-wide multifaceted quality improvement intervention: Rationale and design for a multicenter cluster randomized clinical trial in China. American Heart Journal. 2020;225:44-54.

13. O'Connor C, Adhikari NK, DeCaire K, Friedrich JO. Medical admission order sets to improve deep vein thrombosis prophylaxis rates and other outcomes. Journal of hospital medicine. 2009;4(2):81 - 9.

14. Byrnes MC, Schuerer DJ, Schallom ME, Sona CS, Mazuski JE, Taylor BE, et al. Implementation of a mandatory checklist of protocols and objectives improves compliance with a wide range of evidencebased intensive care unit practices. Critical care medicine. 2009;37(10):2775-81 .

15. Lau BD, Streiff MB, Pronovost PJ, Haut ER. Venous Thromboembolism Quality Measures Fail to Accurately Measure Quality. Circulation. 2018;137(12):1278-84.

16. Borab ZM, Lanni MA, Tecce MG, Pannucci CJ, Fischer JP. Use of computerized clinical decision support systems to prevent venous thromboembolism in surgical patients: a systematic review and meta-analysis. JAMA surgery. 2017;152(7):638 - 45.

17. Haut ER, Lau BD, Kraenzlin FS, Hobson DB, Kraus PS, Carolan HT, et al. Improved prophylaxis and decreased rates of preventable harm with the use of a mandatory computerized clinical decision support tool for prophylaxis for venous thromboembolism in trauma. Archives of surgery. 2012;147(10):901-7.

18. Mosen D, Elliott CG, Egger MJ, Mundorff M, Hopkins J, Patterson R, et al. The effect of a computerized reminder system on the prevention of postoperative venous thromboembolism. Chest. 
2004;125(5):1635-41.

19. Galanter WL, Thambi M, Rosencranz H, Shah B, Falck S, Lin F-J, et al. Effects of clinical decision support on venous thromboembolism risk assessment, prophylaxis, and prevention at a university teaching hospital. American Journal of Health-System Pharmacy. 2010;67(15):1265-73.

20. Soo GC, Wong Doo NC, Burrows J, Ritchie A, Zhang J, Burke R. Improving the adoption of an electronic clinical decision support tool and evaluating its effect on venous thromboembolism prophylaxis prescribing at a Sydney tertiary teaching hospital. Journal of Pharmacy Practice and Research. 2019;49(6):508 - 16.

21. Sliwka D, Fang MC. Venous thromboembolism prophylaxis in the United States: still room for improvement. Journal of general internal medicine. 2010;25(6):484-6.

22. Mahan CE, Spyropoulos AC. Venous thromboembolism prevention: a systematic review of methods to improve prophylaxis and decrease events in the hospitalized patient. Hospital Practice. 2010;38(1):97-108.

23. Kahn SR, Panju A, Geerts W, Pineo GF, Desjardins L, Turpie AG, et al. Multicenter evaluation of the use of venous thromboembolism prophylaxis in acutely ill medical patients in Canada. Thrombosis research. 2007;119(2):145 - 55.

24. Schünemann HJ, Cook D, Grimshaw J, Liberati A, Heffner J, Tapson V, et al. Antithrombotic and thrombolytic therapy: from evidence to application: the Seventh ACCP Conference on Antithrombotic and Thrombolytic Therapy. Chest. 2004;126(3):688S-96S.

25. Hunt BJ. Preventing hospital associated venous thromboembolism. British Medical Journal Publishing Group; 2019.

26. Sharaf RN, Khullar D, Umscheid CA. Health Technology Assessment Centers-an Infrastructure for Health Systems to Translate Evidence into Practice. Journal of General Internal Medicine. 2020:1-4.

27. Karajizadeh M, Zand F, Vazin A, Nasiri M, Sharifian R. Identification and Prioritization of Clinical Decision Support Functionalities Built Within A Computerized Provider Order Entry System. Health Scope. 2021;10(1):e104607.

28. Khammarnia M, Sharifian R, Zand F, Keshtkaran A, Barati O. Designing computerized provider order entry software in Iran: The nurses' and physicians' viewpoints. CIN: Computers, Informatics, Nursing. 2016;34(9):413 - 20.

29. Ruiz-Talero P, Cerón-Perdomo D, Hernández-Flórez C, Gutiérrez-Gómez S, Muñoz-Velandia O. Improving compliance to clinical practice guidelines with a multifaceted quality improvement program for the prevention of venous thromboembolic disease in nonsurgical patients. International Journal for Quality in Health Care. 2020;32(5):319 - 24.

30. George B, Gonzales S, Patel K, Petit S, Franck AJ, Bovio Franck J. Impact of a clinical decisionsupport tool on venous thromboembolism prophylaxis in acutely ill medical patients. Journal of Pharmacy Technology. 2020;36(4):141-7.

31. Roy PM, Rachas A, Meyer G, Le Gal G, Durieux P, El Kouri D, et al. Multifaceted Intervention to Prevent Venous Thromboembolism in Patients Hospitalized for Acute Medical Illness: A Multicenter Cluster- 
Randomized Trial. PLoS One. 2016;11(5):e0154832.

32. Khoury L, Dangodara AA, Lee J-A, Lovejoy M, Amin AN. Implementation of a mandated venous thromboembolism clinical order set improves venous thromboembolism core measures. Hospital Practice. 2014;42(5):89-99.

33. Kucher N, Puck M, Blaser J, Bucklar G, Eschmann E, Lüscher T. Physician compliance with advanced electronic alerts for preventing venous thromboembolism among hospitalized medical patients. Journal of Thrombosis and Haemostasis. 2009;7(8):1291-6.

34. Eijgenraam P, Meertens N, van den Ham R, Ten Cate H, Arina J. The effect of clinical decision support on adherence to thrombosis prophylaxis guidelines in medical patients; A single center experience. Thrombosis research. 2015;135(3):464 - 71.

35. Moja L, Polo Friz H, Capobussi M, Kwag K, Banzi R, Ruggiero F, et al. Effectiveness of a HospitalBased Computerized Decision Support System on Clinician Recommendations and Patient Outcomes: A Randomized Clinical Trial. JAMA Network Open. 2019;2(12):e1917094-e.

36. Sutton RT, Pincock D, Baumgart DC, Sadowski DC, Fedorak RN, Kroeker KI. An overview of clinical decision support systems: benefits, risks, and strategies for success. NPJ Digit Med. 2020;3:17-.

37. De Croon R, Van Houdt L, Htun NN, Štiglic G, Vanden Abeele V, Verbert K. Health Recommender Systems: Systematic Review. J Med Internet Res. 2021;23(6):e18035.

38. Tran TNT, Felfernig A, Trattner C, Holzinger A. Recommender systems in the healthcare domain: state-of-the-art and research issues. Journal of Intelligent Information Systems. 2020.

39. Westerbeek L, Ploegmakers KJ, de Bruijn G-J, Linn AJ, van Weert JCM, Daams JG, et al. Barriers and facilitators influencing medication-related CDSS acceptance according to clinicians: A systematic review. International Journal of Medical Informatics. 2021;152:104506.

\section{Figures}




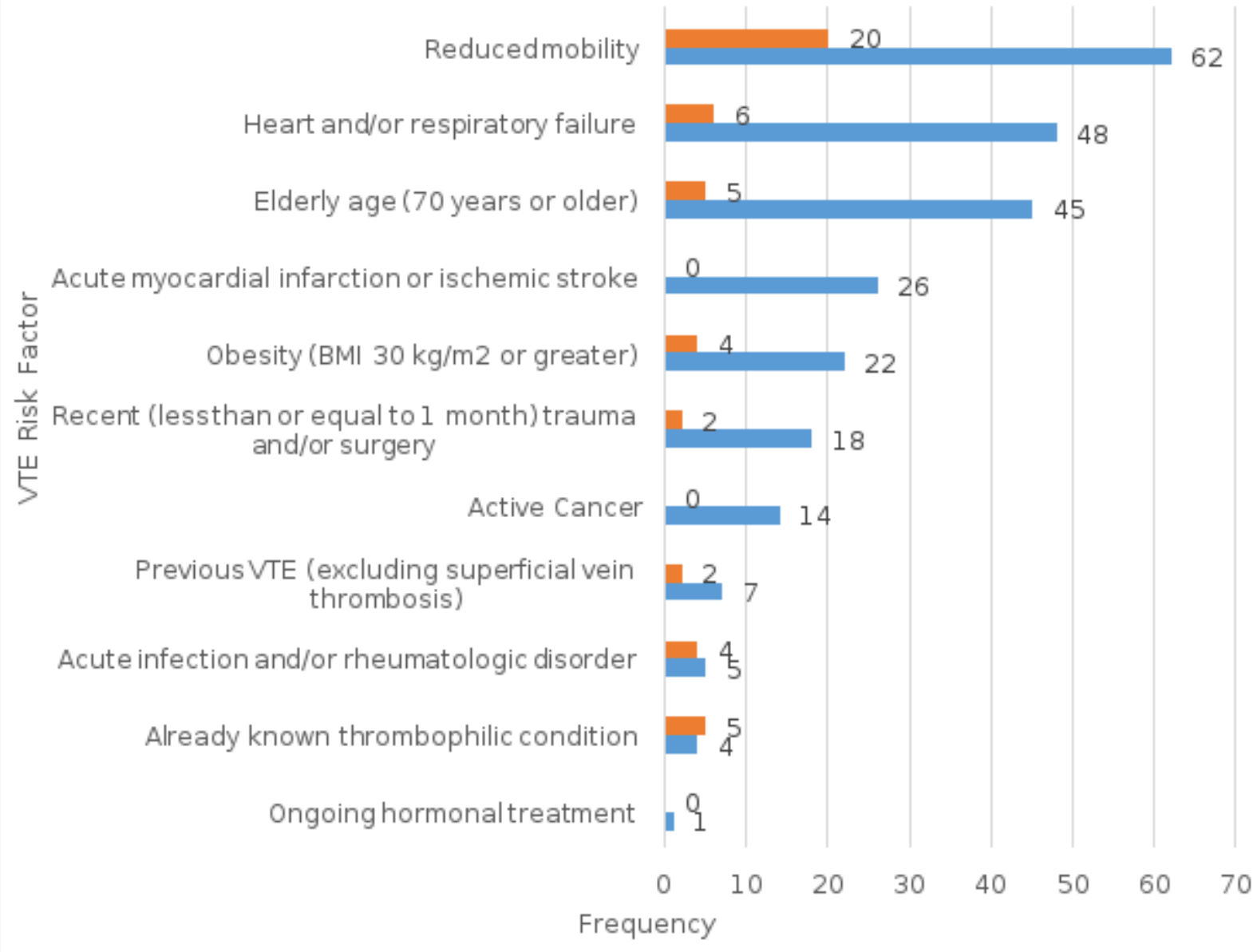

nSum of frequency of eachVTE risk in Post-CDS for recommendationVTE prophylaxis aSum of frequency of eachVTE risk in Pre-CDS for recommendationVTE prophylaxis

\section{Figure 1}

frequency of VTE risk factors in pre and post implementation CDSS for VTE prophylaxis recommendation groups

\section{Supplementary Files}

This is a list of supplementary files associated with this preprint. Click to download.

- SupplementaryFile1.docx

- SupplementrayFile2.docx

- SupplementryFile3.docx 